\title{
Laccase biosensors based on different enzyme immobilization strategies for phenolic compounds determination
}

\author{
E. Casero ${ }^{\mathrm{a}}$, M.D. Petit-Domínguez ${ }^{\mathrm{a}}$, L. Vázquez ${ }^{\mathrm{b}}$, I. Ramírez-Asperilla $^{\mathrm{a}}$, A.M. Parra- \\ Alfambra $^{\mathrm{a}}$, F. Pariente ${ }^{\mathrm{a}}$, E. Lorenzo ${ }^{\mathrm{a}^{*}}$
}

${ }^{a}$ Departamento de Química Analítica y Análisis Instrumental. Facultad de Ciencias. c/ Francisco Tomás y Valiente, $N^{\circ} 7$.

${ }^{b}$ Instituto de Ciencia de Materiales de Madrid (CSIC). c/ Sor Juana Inés de la Cruz $N^{o} 3$. Campus de Excelencia de la Universidad Autónoma de Madrid. 28049 Madrid. Spain.

e-mail: encarnacion.lorenzo@uam.es

\begin{abstract}
Different enzyme immobilization approaches of Trametes versicolor laccase (TvL) onto gold surfaces and their influence on the performance of the final bioanalytical platforms are described. The laccase immobilization methods include: i) direct adsorption onto gold electrodes (TvL/Au), ii) covalent attachment to a gold surface modified with a bifunctional reagent, 3,3'-Dithiodipropionic acid di ( $\mathrm{N}$-succinimidyl ester) (DTSP), and iii) integration of the enzyme into a sol-gel 3D polymeric network derived from (3-mercaptopropyl)-trimethoxysilane (MPTS) previously formed onto a gold surface (TvL/MPTS/Au). The characterization and applicability of these biosensors are described. Characterization is performed in aqueous acetate buffer solutions using atomic force microscopy (AFM), providing valuable information concerning morphological data at the nanoscale level.

The response of the three biosensing platforms developed, TvL/Au, TvL/DTSP/Au and TvL/MPTS/Au, is evaluated in the presence of hydroquinone (HQ), used as a phenolic enzymatic substrate. All systems exhibit a clear electrocatalytic activity and HQ can be amperometrically determined at $-0.10 \mathrm{~V}$ versus $\mathrm{Ag} / \mathrm{AgCl}$. However, the performance of biosensors -evaluated in terms of sensitivity, detection limit, linear
\end{abstract}


response range, reproducibility and stability- depends clearly on the enzyme immobilization strategy, which allows establishing its influence on the enzyme catalytic activity.

Keywords: amperometric biosensors, enzyme immobilization, Trametes versicolor laccase, phenolic compounds, atomic force microscopy. 


\section{Introduction}

Development of amperometric biosensors based on enzyme immobilization onto electrode surfaces in such way that they retain their fully activity and stability has become one of the most important issues in several fields ranging from environmental analysis to clinical diagnosis.

In this sense, nowadays, in nutritional literature, a high effort is devoted to study several aspects of free radicals, a type of unstable compounds produced during metabolic processes. An excess of free radicals in the human body, which can be caused by pollution, sunlight or smoke of cigarettes overexposure, promotes oxidative damage in cells, accelerating the aging process. Among the different compounds with antioxidant properties, polyphenols play a protective role against human diseases associated with oxidative stress. They are commonly present in fruits, legumes, vegetables and several beverages. Accordingly, during the last years, numerous studies aimed at evaluating the antioxidant capacity of natural products, such as wines, teas and herbal infusions, have been reported. In particular, special attention has been paid to the development of new methodologies, such as amperometric biosensors based on tyrosinase, peroxidase or laccase enzymes, which can be employed as alternative methods for total polyphenolic content determination in foods [1-10].

Laccases, which have been employed in a less extent than tyrosinases for phenols quantification, are a type of copper-containing oxidoreductases produced by numerous plants, fungi, and bacteria. In particular, Trametes versicolor laccase, TvL, (dimensions of $7.0 \mathrm{~nm} \times 5.0 \mathrm{~nm} \times 5.6 \mathrm{~nm})[11]$ is a fungal laccase containing four copper ions located in sites denoted as T1, T2 and T3 in the active center of the enzyme [12-14]. Among laccases from different sources, there are remarkable differences such as molecular weight, substrate specificity and optimum $\mathrm{pH}$, but they have in common the 
capability to oxidize benzenediols/phenols to the corresponding phenones with the concomitant reduction of oxygen. Thus, the detection of polyphenolic compounds can be based on measuring the reduction current of quinones generated as products of the enzymatic reaction.

The performance of enzyme-based amperometric biosensors depends on several factors, mainly on the catalytic activity preservation after the enzyme immobilization onto solid electrodes. Since the immobilization process can produce an alteration of the three-dimensional conformation of the enzyme that leads to a diminution of the catalytic activity, an adequate choice of the immobilization strategy plays a crucial role in the biosensor design and performance. In the particular case of laccase-based biosensors, an extensive research effort has been addressed to immobilize laccase on surfaces by different immobilization strategies in order to design biosensors with a wide range of applications $[15,16]$. In this sense, laccase immobilization has been performed in the literature on different solid supports (glassy carbon, carbon paste, platinum, gold) using immobilization strategies such as direct adsorption, covalent binding, entrapment in polymeric membranes or gels and cross-linking procedures. Recent advances in this field are related to the development of new support matrix as suitable platforms (most of them including nanomaterials) for enzymes immobilization, which lead to biosensors with improved analytical properties. So, in recent works, laccase has been immobilized by direct adsorption, covalent binding or entrapment onto: epoxy resin membranes [9], mesoporous materials with well-controlled pore structures [10, 17], multi-walled carbon nanotubes paste electrodes [18], nanocomposites formed by chitosan and carbon nanotubes [19], copper-containing ordered mesoporous carbon chitosan matrix [8], pyrenehexanoic acid-modified hierarchical carbon microfibers/carbon nanotubes composite electrodes [16], polyvinyl alcohol photopolymers [20], sol-gel matrix of 
diglycerylsilane [21], 3-mercaptopropionic acid self-assembled monolayer modified gold electrodes [22], cysteine self-assembled monolayer and quantum dots modified gold electrodes [23], nanocomposites of silver nanoparticles and zinc oxide nanoparticles electrochemically deposited onto gold electrodes [24], platinum nanoparticles and reduced graphene composites deposited onto screen printed electrodes [25], polyethyleneimine coated gold-nanoparticles modified glassy carbon electrodes [26], multiwalled carbon nanotubes and polypyrrole electrocodeposited onto platinum electrodes [27].

In this paper, we describe the development and characterization of three different laccase, from Trametes versicolor (TvL), based biosensors for polyphenols determination. Among the different available strategies to achieve the enzyme immobilization onto gold surfaces, we have focused our attention on: i) direct adsorption of TvL onto a gold substrate, ii) covalent bonding to a self-assembled monolayer (SAM) and iii) encapsulation into a sol-gel matrix. The morphological characteristics at the nanometer level of the developed biosensors were obtained by atomic force microscopy (AFM) technique. The influence of the enzyme immobilization strategy on the analytical performance of the resulting biosensors was evaluated using hydroquinone as a model phenolic substrate.

\section{Experimental Section}

\subsection{Reagents and solutions}

Laccase (EC 1.1.3.4) from Trametes versicolor (TvL), lyophilized powder containing 27.9 U/mg solid, was obtained from the Sigma Chemical Co. (St. Louis, MO). Stock enzyme solution was prepared dissolving $35.7 \mathrm{mg}$ of the TvL lyophilized powder in $250 \mu \mathrm{L}$ of $0.1 \mathrm{M}$ acetic/acetate buffer solution $\mathrm{pH} 4.5$, aliquoted $(10 \mu \mathrm{L})$ and 
stored at $-30^{\circ} \mathrm{C}$. Under these conditions, the enzymatic activity remains stable for several weeks. Hydroquinone (HQ), 3,3'-Dithiodipropionic acid di (N-succinimidyl ester) (DTSP), dimethyl-sulfoxide (DMSO) and (3-mercaptopropyl)-trimethoxysilane (MPTS) were also purchased from Sigma Chemical Co. and used as received. Stock solutions of HQ (1 mM) were freshly prepared. Other chemicals used in this work, such as methanol, hydrochloric acid and sodium acetate were used as received without additional purification steps. Sodium acetate was employed for the preparation of acetic/acetate buffer solutions $(0.1 \mathrm{M}, \mathrm{pH} 4.5)$ Water was purified with a Millipore Milli-Q-System. All solutions were prepared just prior to use.

\subsection{Experimental techniques}

\subsubsection{Atomic force microscopy measurements}

Supports employed for laccase immobilization in AFM measurements consist of glass substrates $\left(1.1 \times 1.1 \mathrm{~cm}^{2}\right)$ covered with a chromium layer $(1-4 \mathrm{~nm}$ thick $)$ on which a gold layer (200-300 nm thick) was deposited (Metallhandel Schröer GmbH, Lienen, Germany). The AFM measurements were performed with a Nanoscope IIIa equipment (Bruker) operating in intermittent contact and under buffer conditions. Sharp silicon nitride levers (Bruker) with a nominal force constant close to $0.32 \mathrm{~N} / \mathrm{m}$ were employed. These cantilevers have a silicon tip with a nominal tip radius of about $2 \mathrm{~nm}$. Imaging soft matter under liquid environment with this sharp tip requires an extreme care to keep low the applied tip load since if this is not the case the soft sample feature could be pierced or deformed by the tip. Accordingly, extreme low free amplitudes (around 0.2$0.3 \mathrm{~V})$ were employed. The images were obtained in 3-6 minutes and were composed by 512 x 512 pixels. The thickness of the MPTS layer was estimated in air conditions with silicon cantilevers through force curve analysis.

\subsubsection{Electrochemical measurements}


Cyclic voltammetric and amperometric studies were carried out with an Ecochemie Autolab PGSTAT12 system (Utrecht, The Netherlands). The electrochemical experiments were carried out in a three-compartment electrochemical cell with standard taper joints so that all compartments could be hermetically sealed with Teflon adapters. A gold electrode was used as working electrode. A large-area coiled platinum wire was employed as a counter electrode. All potentials were reported against a $\mathrm{Ag} / \mathrm{AgCl}$ reference electrode without taking into account the liquid junction.

\subsection{Procedures}

\subsubsection{Preparation of the electrochemical biosensing platforms}

Prior to each experiment, gold electrodes were polished with $1 \mu \mathrm{m}$ diamond paste (Buehler) and rinsed with water.

The TvL/Au electrochemical biosensing platform was developed by placing $5 \mu \mathrm{L}$ of the stock enzyme solution onto the electrode surface. After air-drying, the modified electrodes were washed with water to remove any weakly bound material.

The TvL/DTSP/Au was developed by immersing a gold substrate for $3 \mathrm{~h}$ at room temperature in a solution of DTSP (1.0 mM in DMSO). The corresponding monolayer containing the active succinimidyl esters was rinsed in DMSO and water. Subsequently, $5 \mu \mathrm{l}$ of TvL stock solution was placed onto the modified surface (DTSP/Au) leading to a covalent binding between the enzyme and the succinimidyl esters. After air-drying, the biosensor was washed with water to remove any weakly bound enzyme.

Finally, the development of the TvL/MPTS/Au biosensing platform requires the previous formation of a sol gel network. In a first step, the sol-gel solution was prepared mixing $200 \mu \mathrm{L}$ of MPTS with $600 \mu \mathrm{L}$ of water, $600 \mu \mathrm{L}$ of methanol and $200 \mu \mathrm{L}$ of 0.1 $\mathrm{M} \mathrm{HCl}$. This solution was sonicated using an Ultrasons bath (P-Selecta) for $30 \mathrm{~min}$ at room temperature. Subsequently, the solution was stored for three hours at room 
temperature. Afterwards, the gold surface was immersed in the MPTS sol-gel solution, prepared as described above, for 1 hour. MPTS sol chemisorbs on the polycrystalline Au electrode forming a 3D silicate network. The resulting MPTS sol-modified electrode was thoroughly rinsed with water to remove the physically adsorbed MPTS sol and finally $5 \mu \mathrm{L}$ of the TvL stock solution were dropped on this surface. After air-drying, the biosensor was washed with water to remove any weakly bound enzyme.

The three different enzyme immobilization approaches employed for biosensors preparation are summarized in scheme $1 \mathrm{~A}$.

\subsubsection{AFM measurements}

Gold supports employed for AFM measurements were previously annealed for 2 min in a gas flame in order to obtain $\mathrm{Au}$ (111) terraces and imaged in buffer solution to assess that the surface was flat and clean prior to enzyme immobilization. The images, before and after TvL immobilization, were taken with different cantilevers in order to ensure that the imaged structures were not due to tip artefacts.

\section{Results and discussion}

\subsection{Immobilization of TvL onto gold surfaces by different approaches:} characterization by AFM.

A critical step in developing biosensors is the enzyme immobilization onto the transducer. Physical adsorption is a rapid and easy way to immobilize enzymes on gold, which is a rather interesting material for bioelectronic devices. However, it may result in low stability of the enzyme on the electrode; thus a stronger linkage is sometimes required for biosensor applications. One of the approaches employed successfully to achieve strong immobilization of enzymes on gold surfaces consists of chemical modification of the electrode surface for favoring specific interactions with the enzyme molecules. Entrapping enzymes into polymeric structures or hydrogels allows a higher 
concentration of enzyme on the electroactive surface, but the background current is increased. In this work, we have developed and compared three different strategies, based on the principles mentioned above, to immobilize Trametes versicolor laccase: i) direct adsorption onto a gold electrode (TvL/Au), ii) covalent attachment on a previously functionalized gold surface with an adequate self-assembled monolayer (SAM) (TvL/DTSP/Au) and iii) encapsulation of TvL onto a sol-gel matrix (TvL/MPTS/Au). In the former case (TvL/Au), the interaction responsible of the enzyme immobilization is unspecific adsorption. In contrast, in the second approach we have used a well-established strategy to bind covalently proteins. This approach is based on the covalent attachment of proteins containing lysine residues, through acylation of free primary or secondary aliphatic groups, to gold surfaces modified with a bifunctional reagent containing succinimide functionalities, in particular, 3,3' Dithiodipropionic acid di (N-succinimidyl ester) (DTSP). Since DTSP adsorbs onto gold surfaces through the disulfide group, the terminal succinimidyl groups are exposed to the solution allowing further covalent immobilization of amine-containing biomolecules, such as laccase from Trametes versicolor (TvL/DTSP/Au). Finally, the third approach is based on TvL encapsulation on a sol-gel 3D polymeric network derived from (3-mercaptopropyl)-trimethoxysilane (MPTS) previously formed onto a gold surface. MPTS presents the advantage of forming a 3D polymeric network containing a large number of thiol tail groups distributed throughout its structure that enable its anchoring onto gold surfaces. Moreover, this matrix provides a biocompatible environment that preserves the catalytic activity of TvL after its immobilization and allows the incorporation of a high amount of enzyme, which is expected to improve the sensitivity of the final biosensing device. Since the performance of the developed biosensors is closely related to several factors including the conformation, distribution 
and morphology of the immobilized enzyme onto the surface, performing an AFM characterization of each system becomes essential.

Figure 1A shows a $3.5 \times 3.5 \mu \mathrm{m}^{2}$ image of the gold surface after deposition of laccase by direct adsorption. Clearly, the characteristic micro-granular structure of the underlying gold surface is observed. The bright spots observed in the image correspond to laccase aggregates that usually tend to be formed along the steps and the micro-grain boundaries of the gold surface. Characteristic $0.5 \times 0.5 \mu \mathrm{m}^{2}$ images obtained on the surface of a flat micro-grain (figure 1B) reveal that the whole surface is covered by a compact layer of globular structures with characteristic lateral sizes in the 5-8 $\mathrm{nm}$ range. In a higher resolution image $\left(75 \times 75 \mathrm{~nm}^{2}\right)$ of this layer (inset of figure $\left.1 \mathrm{~A}\right)$ the globular morphology and size of these structures are better observed. As these structures were not present in the as-prepared gold surface (data not shown), and their lateral dimensions are consistent with those of the laccase molecule, we can identify them as those of the laccase deposit. In fact, the brighter (i.e., higher) spots observed in figures 1A and 1B correspond either to single proteins adsorbed on top of the laccase monoloyer (with heights in the 6-7 $\mathrm{nm}$ range) or to laccase aggregates displaying larger height values (above $10 \mathrm{~nm}$ ).

The AFM analysis of the TvL/DTSP/Au system (figures 1C and 1D) shows a similar behavior than that observed on the previous system (TvL/Au). Perhaps, the most noticeable difference lies in the fact that it seems that laccase tends to aggregate mainly at the micro-grain boundaries as figure $1 \mathrm{C}$ shows. Once more, the images taken on flat micro-grains (figure 1D) display a compact laccase monolayer, where the laccase structures show lateral sizes in the 5-7 $\mathrm{nm}$ range (see inset of figure 1C). Again, some structures 6-8 nm high, are imaged on top of the laccase monolayer, which correspond to laccase structures deposited on top of the underlying monolayer. 
Finally, when laccase is included in a 3D polymeric network, the resulting platform (TvL/MPTS/Au) presents some morphological differences with respect to the two previous ones. In order to better interpret the AFM images obtained in this system, it is convenient to first characterize the MPTS/Au system. Its surface morphology as imaged by $\mathrm{AFM}$ is shown in figures $2 \mathrm{~A}$ and $2 \mathrm{~B}$. The larger image (figure $2 \mathrm{~A}$ ) shows a flatter surface as the grooves between micro-grains are to some extent blurred or less marked, probably due to the deposit of the polymer layer at these locations. However, some sort of surface roughness is evident in both figures $2 \mathrm{~A}$ and $2 \mathrm{~B}$, although it becomes clear that the MPTS/Au surface morphology at the nano-scale is different from those obtained in laccase-containing systems (compare figure 2B with figures $1 \mathrm{~B}$ or 1D).

The previous data will be useful to interpret those obtained from the AFM images of the TvL/MPTS/Au surface (figures $3 \mathrm{~A}$ and 3B). In this case, the micro-granular structure of the gold substrate is also blurred or dimmed as the grain boundaries are not so clearly observed now (figure 3A). This fact agrees with the observations already reported on the MPTS/Au surface [28]. It is worth to note that the surface of the flat grains is more homogeneous as quite less three dimensional laccase aggregates are imaged (figure 3B). However, the high resolution imaging (inset of figure 3A) shows that the laccase layer is less compact (more porous) than in the previous cases, where it was physically adsorbed or covalently bonded. This larger porosity together with tip convolution effects, leads to wider globular structures (in the 5-12 $\mathrm{nm}$ range) than those previously observed for the other two systems where laccase formed a quite compact monolayer. This fact can be related to the presence of the MPTS layer itself. This soft polymeric layer can swell when immersed in liquid environment as in this case. Under this condition, the sharp tip could pierce this quite soft layer and image laccase structures located at different depths inside the MPTS layer. In this sense, we have tried 
to estimate the thickness of the MPTS layer. For this purpose, we have taken different force curves in air with the same tip on the Au surface and the MPTS/Au surface.

In figure $2 \mathrm{C}$ are displayed characteristic force curves taken on the gold surface and at three different spots of the MPTS/Au sample. The steepest slope is observed for the Au sample because of its stiffer character. For the MPTS/Au sample, there is first a curved region, which corresponds to the softer MPTS layer, followed by a straight regime with a slope similar to that measured on Au. This last regime then corresponds to the point where the soft MPTS layer has been pierced and the underlying Au surface has been reached. From the lateral shift at this point between the MPTS/Au curve and that measured on $\mathrm{Au}$, MPTS thickness values ranging from $20 \mathrm{~nm}$ up to $75 \mathrm{~nm}$ are obtained. Therefore, as one would expect for a 3D network, the MPTS layer could stack a large number of laccase structures at different depths, which would be consistent with the images obtained for the TvL/MPTS/Au system. Based on the above results, it seems clear that the third strategy allows the immobilization of a great number of laccase molecules per unit of geometric area than those based on either direct adsorption or covalent binding to a SAM-modified gold electrode.

\subsection{Evaluation of the biosensor response.}

Laccase catalyzes the oxidation of benzenediols with concomitant reduction of $\mathrm{O}_{2}$ to $\mathrm{H}_{2} \mathrm{O}$ [29]. In the biosensing platforms developed in this work, hydroquinone was selected as a model phenolic substrate. According to scheme 1B, laccase catalyzes the oxidation of hydroquinone to quinone through its active center T1 acting the molecular oxygen as final electron acceptor, which is reduced to water [12]. The quinone enzymatically generated is reduced to hydroquinone at the electrode surface, giving rise to an electrocatalytic current. Thus, the concentration of hydroquinone, or other 
phenolic substrates, can be determined by monitoring this electrocatalytic current.

In order to assess whether the developed laccase based biosensors present catalytic activity towards phenolic compounds and to evaluate the influence of the enzyme immobilization strategy on the analytical performance of the resulting devices, its cyclic voltammetric response in the absence and in the presence of hydroquinone was obtained.

Hydroquinone presents electrochemical response at gold electrode, thus the first step was to study its response under the same experimental condition than those employed for the biosensor. Figure 4A displays the cyclic voltammogram of a bare $\mathrm{Au}$ electrode in the presence of hydroquinone in $0.1 \mathrm{M}$ acetate buffer $(\mathrm{pH} 4.5)$ solution. As can be observed, when the potential was cycled from $+0.18 \mathrm{~V}$ (where no faradaic current is observed) towards positive values and then reversed until $-0.5 \mathrm{~V}$, a pair of redox peaks with a formal potential of $+0.20 \mathrm{~V}$, ascribed to the hydroquinone/quinone redox couple, is observed. The peak potential separation $\left(\Delta \mathrm{E}_{\mathrm{p}}=400 \mathrm{mV}\right)$ is larger than the typical value for two-protons / two-electrons redox reaction, indicating a charge transfer resistance between redox species and electrode surface. If the potential is swept from $+0.18 \mathrm{~V}$ towards negative values and then reversed until $+0.6 \mathrm{~V}$ (figure 4B), no reduction peak appears in the cathodic scan, indicating the absence of quinones or other oxidants in the initial solution. However, the oxidation of the hydroquinone is evident in the anodic scan.

Figure $4 \mathrm{C}$ shows the cyclic voltammogram response for the TvL/Au biosensing platform in the presence (a) and in the absence (b) of hydroquinone in $0.1 \mathrm{M}$ acetate buffer solution, from $-0.5 \mathrm{~V}$ to $+0.65 \mathrm{~V}$ at $10 \mathrm{mV} / \mathrm{s}$. The potential sweep was carried out at the same conditions as in figure 4B. In the presence of hydroquinone (scan a of figure 4C), it can be observed that in the anodic scan there are not oxidation peaks, indicating 
that hydroquinone was not present near the electrode since the enzymatic reaction was taking place. In contrast, in the cathodic scan, since quinone is generated as product of the enzymatic reaction, a clear response ascribed to its electrocatalytic reduction was observed. Obviously, in the absence of hydroquinone (scan b of figure 4C) no peaks were obtained. For both TvL/DTSP/Au and TvL/MPTS/Au platforms, similar cyclic voltammograms to those displayed in figure $4 \mathrm{C}$ were obtained (Figure 1S).

The optimal response of biosensors depends on several factors including the degree of catalytic activity preservation after the enzyme immobilization process and operational parameters such as the working $\mathrm{pH}$ and the applied potential in chronoamperometric measurements. Concerning operational parameters, we have assayed $\mathrm{pH}$ values between 4 and 7 (data not shown). A $\mathrm{pH}$ in the 4-5 range was selected since this gives the optimum response, according to the optimum $\mathrm{pH}$ value for the enzyme [20]. On the other hand, from figure 4C (where the cathodic wave ascribed to the electrocatalytic reduction of p-quinone to hydroquinone appears at $-0.07 \mathrm{~V}$ ) a potential value of $-0.10 \mathrm{~V}$ was selected to carry out the chronoamperometric experiments, since it is adequate to provide a good intensity signal, minimizing interferences due to other redox compounds. Thus, chronoamperometric measurements of TvL/Au, TvL/DTSP/Au and TvL/MPTS/Au platforms for different HQ concentrations were carried out by posing the biosensor at a step-potential from +0.18 $\mathrm{V}$, where no redox process is occurring, to $-0.10 \mathrm{~V}$, where reduction of the quinones generated as products of the enzymatic reaction was assured. From the chronoamperometric response obtained for the developed biosensors in the presence of increasing HQ concentrations (as an example data for TvL/Au system are displayed in figure $2 \mathrm{~S}$ ), it can be concluded that a steady state current is reached after 50s. 
Therefore, the current measured at this time was plotted as a function of the concentration of HQ in solution (figure 3S) for TvL/Au (curve A), TvL/DTSP/Au (curve B) and TvL/MPTS/Au (curve C) biosensors. The linear concentration range for each biosensor was described by the following linear regression equations: $I_{s s}(\mu A)=2.29 C(m M)+0.0014 \quad(R=0.992) \quad$ for $\quad T v L / A u, \quad I_{s s}(\mu A)=2.53 C(m M)+0.0015$ $(\mathrm{R}=0.990)$ for $\quad \mathrm{TvL} / \mathrm{DTSP} / \mathrm{Au}, \quad$ and $\quad \mathrm{I}_{\mathrm{ss}}(\mu \mathrm{A})=2.84 \mathrm{C}(\mathrm{mM})+0.0048 \quad(\mathrm{R}=0.990) \quad$ for TvL/MPTS/Au. The analytical properties of the developed biosensors, such as linear concentration range, sensitivity, detection limit and reproducibility were evaluated, and are displayed in Table 1. As can be observed, the sensitivity, calculated as the slope of

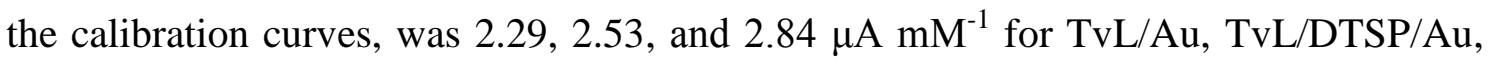
and TvL/MPTS/Au, respectively. Concerning the detection limit, calculated as the ratio between three times the standard deviation of the blank signal and the sensitivity, values of $0.91,0.89$, and $0.25 \mu \mathrm{M}$ were obtained. As can be concluded from these data, biosensors based on direct adsorption and covalent binding show similar linear concentration ranges that are narrower than the corresponding to sol-gel based one. Regarding sensitivity and detection limit, they are higher and lower respectively, in the case of sol-gel based platforms. These results are consistent with the fact that this approach allows to incorporate a large number of laccase structures at different depths as it was concluded from AFM images. The reproducibility was evaluated from the RSD for five different measurements of $7 \mu \mathrm{M}$ of hydroquinone, yielding similar values for the biosensors based on the three different immobilization strategies (around 3\%).

Thus, if we compare the results obtained for the biosensor based on three dimensional sol-gel network with the results obtained for both dimensional biosensors, based on direct adsorption or covalent binding, we can affirm that the three strategies allowed to prepare very reproducible final biosensing devices. Nevertheless, as one 
would expect and according to the literature, the three dimensional sol-gel network allows the incorporation of a higher amount of enzyme, which improves the sensitivity of the final biosensing device without lost of reproducibility.

In order to compare the analytical properties obtained for the three developed biosensors with those of other similar biosensors, we have also included in table 1 data concerning linear concentration range, sensitivity, detection limit and reproducibility of several laccase-based biosensors reported in the literature $[3,4,8-10,17,19-21,25-27$, 30-35]. It is important to highlight that, in general, a direct comparison of the performance of the different biosensors is not straightforward since they employ different detection strategies, they determine different phenolic substrates and they are developed using different sources of enzymes. However, although a high variability of data is observed for each analytical parameter (due to the different nature of biosensors displayed in table 1), it can be concluded that most of the analytical properties of the biosensors designed by us are comparable or even better than those reported in the literature for other laccase-based biosensors. It is important to highlight that some of the biosensors summarized in the table include nanomaterials, which is not our case. Moreover, an important point to take into account is that related to the simplicity of the biosensor fabrication process. In this sense, the three biosensors designed in the present work involve an easy fabrication process, allowing to obtain similar or better analytical parameters than those showed in the table, most of them obtained with more complicated methods.

Finally, since one of the most critical issues for an enzyme immobilization approach is to avoid the leaking of the immobilized enzyme to the solution and to preserve its catalytic activity, the stability of TvL/Au, TvL/DTSP/Au and TvL/MPTS/Au biosensors was also evaluated by recording successive cyclic 
voltammograms in $0.1 \mathrm{M}$ acetate buffer $(\mathrm{pH} 4.5)$ solution containing $100 \mu \mathrm{M}$ hydroquinone. From these measurements, displayed in figure 1S, it can be concluded that about $89 \%, 96 \%$ and $99 \%$ of the initial current response was retained after 50 measurements for TvL/Au, TvL/DTSP/Au and TvLMPTS/Au, respectively. In order to study the storage stability of the three developed biosensors, additional experiments were carried out. Biosensors were stored at $4^{\circ} \mathrm{C}$, when not in use. Biosensors maintain their initial response during the first week of storage, while after six weeks TvL/Au, TvL/DTSP/Au and TvL/MPTS/Au retain $87 \%, 93 \%$ and $97 \%$ of the initial current response, indicating that the developed biosensing platforms, particularly the based on sol-gel technology, present excellent storage stability. The results indicate that specially in the case of the TvL/MPTS/Au biosensor, the enzyme was immobilized in a friendly and biocompatible environment.

\section{Conclusions}

In this paper, the influence of different enzyme immobilization strategies on the performance of TvL biosensing platforms is studied. The three methods selected for the immobilization process are based on different interactions between the enzyme and the surface: i) unspecific adsorption, ii) covalent binding and iii) inclusion in a sol gel matrix. In presence of a phenolic compound, the biosensing platforms exhibit electrocatalytic activity, and hydroquinone can be amperometrically determined at -0.10 $\mathrm{V}$ versus $\mathrm{Ag} / \mathrm{AgCl}$. The biosensor based on sol-gel technology exhibits a lower detection limit and a wider linear concentration range, when compared with biosensors based on direct adsorption and covalent binding, respectively. In this sense, this relative biosensing performance enhancement is explained by the incorporation of a higher amount of enzyme, which is enabled by the three dimensional sol-gel network. In 
addition, the sol-gel network provides with a biocompatible and friendly environment to enzyme encapsulation, leading to biosensors with an excellent stability.

\section{Acknowledgments}

This work has been supported by Comunidad Autónoma de Madrid (project No. S2009/PPQ-1642, AVANSENS) and Ministerio de Ciencia e Innovación (projects No. CTQ2008-05775 and FIS2012-38866-C05-05). 


\section{REFERENCES}

[1] S.A.S.S. Gomes, J.M.F. Nogueira, M.J.F. Rebelo, Biosens. Bioelectron. 20 (2004) 1211-1216.

[2] F. Vianello, S. Ragusa, M.T. Cambria, A. Rigo, Biosens. Bioelectron. 21 (2006) 2155-2160.

[3] M.L. Mena, V. Carralero, A. González-Cortés, P. Yáñez-Sedeño, J.M. Pingarrón, Electroanalysis 17 (2005) 2147-2155.

[4] A.I. Yaropolov, S.V. Shleev, O.V. Morozova, E.A. Zaitseva, G. Marko-Varga, J. Emneus, L. Gorton, J. Anal. Chem. 60 (2005) 553-557.

[5] H. Tang, W. Zhang, P. Geng, Q. Wang, L. Jin, Z. Wu, M. Lou, Anal. Chim. Acta. 562 (2006) 190-196.

[6] Y. Zhang, G. Zeng, L. Tang, D. Huang, X. Jiang, Y. Chen, Biosens. Bioelectron. 22 (2007) 2121-2126.

[7] Y. Tan, W. Deng, Y. Li, Z. Huang, Y. Meng, Q. Xie, M. Ma, S. Yao, J. Phys. Chem. B 114 (2010) 5016-5024.

[8] X. Xu, M. Guo, P. Lu, R. Wang, Mater. Sci. Eng. C-Mat 30 (2010) 722-729.

[9] S. Chawla, R. Rawal, Shabnam, R.C. Kuhad, C.S. Pundir, Anal. Methods 3 (2011) 709-714.

[10] T. Shimomura, T. Itoh, T. Sumiya, T. Hanaoka, F. Mizukami, M. Ono, Sensor. Actuat. B-Chem. 153 (2011) 361-368.

[11] T. Bertrand, C. Jolivalt, P. Briozzo, E. Caminade, N. Joly, C. Madzak, C. Mougin, Biochemistry 41 (2002) 7325-7333.

[12] S. Shleev, J. Tkac, A. Christenson, T. Ruzgas, A.I. Yaropolov, J.W. Whittaker, L. Gorton, Biosens. Bioelectron. 20 (2005) 2517-2554. 
[13] F. Xu, A.E. Palmer, D.S. Yaver, R.M. Berka, G.A. Gambetta, S.H. Brown, E.I. Solomon, J. Biol. Chem. 274 (1999) 12372-12375.

[14] S. Shleev, A. Christenson, V. Serezhenkov, D. Burbaev, A. Yaropolov, L. Gorton, T. Ruzgas, Biochem. J. 385 (2005) 745-754.

[15] N. Durán, M.A. Rosa, A. D’Annibale, L. Gianfreda, Enzyme Microb. Tech. 31 (2002) 907-931.

[16] C. Gutiérrez-Sánchez, W. Jia, Y. Beyl, M. Pita, W. Schuhmann, A.L. De Lacey, L. Stoica, Electrochim. Acta 82 (2012) 218-223.

[17] X. Xu, P. Lu, Y. Zhou, Z. Zhao, M.Guo, Mater. Sci. Eng. C-Mat 29 (2009) 21602164.

[18] T.M.B.F. Oliveira, M.F. Barroso, S. Morais, P. de Lima-Neto, A.N. Correia, M.B.P.P.Oliveira, C. Delerue-Matos, Talanta 106 (2013) 137-143.

[19] M. Diaconu, S.C. Litescu, G.L. Radu, Microchim. Acta 172 (2011) 177-184.

[20] P. Ibarra-Escutia, J. Juarez-Gómez, C. Calas-Blanchard, J.L. Marty, M.T. Ramírez-Silva, Talanta 81 (2010) 1636-1642.

[21] M.R. Montereali, L.D. Seta, W. Vastarella, R. Pilloton, J. Mol. Catal. B-Enzym. 64 (2010) 189-194.

[22] F. Vianello, A. Cambria, S. Ragusa, M.T. Cambria, L. Zennaro, A. Rigo, Biosens. Bioelectron. 20 (2004) 315-321.

[23] Z. Wang, Q. Xu, J.H. Wang, Q. Yang, J.H. Yu, Y.D. Zhao, Microchim. Acta 165 (2009) 387-392.

[24] S. Chawla, R. Rawal, D. Kumar, C.S. Pundir, Anal. Biochem. 430 (2012) 16-23.

[25] S.A.V. Eremia, I. Vasilescu, A. Radoi, S.C. Litescu, G.L. Radu, Talanta 110 (2013) 164-170. 
[26] D. Brondani, B. de Souza, B.S. Souza, A. Neves, I.C. Vieira, Biosens. Bioelectron. 42 (2013) 242-247.

[27] I. Cesarino, H.V. Galesco, F.C.Moraes, M.R.V. Lanza, S.A.S. Machado, Electroanalysis 25 (2013) 394-400.

[28] A.M. Parra-Alfambra, E. Casero, M.D. Petit-Domínguez, M. Barbadillo, F. Pariente, L. Vázquez, E. Lorenzo, Analyst 136 (2011) 340-347.

[29] A.I. Yaropolov, O.V. Skorobogatko, S.S. Vartanov, S.D. Varfolomeyev, Appl. Biochem. Biotech. 49 (1994) 257-280.

[30] A. Jarosz-Wilkolazka, T. Ruzgas, L. Gorton, Talanta 66 (2005) 1219-1224.

[31] S.C. Litescu, S.A.V. Eremia, A. Bertoli, L. Pistelli, G.L. Radu, Anal. Lett. 43 (2010) 1089-1099.

[32] M. Diaconu, S.C. Litescu, G.L. Radu, Sensor. Actuat. B-Chem. 145 (2010) 800806.

[33] M. ElKaoutit, I. Naranjo-Rodriguez, K.R. Temsamani, M.P. Hernández-Artiga, D. Bellido-Milla, J.L. Hidalgo-Hidalgo de Cisneros, Food Chem. 110 (2008) 10191024.

[34] B. Haghighi, L. Gorton, T. Ruzgas, L.J. Jönsson, Anal. Chim. Acta 487 (2003) 314.

[35] Y. Liu, X. Qu, H. Guo, H. Chen, B. Liu, S. Dong, Biosens. Bioelectron. 21 (2006) 2195-2201. 


\section{FIGURES}

A

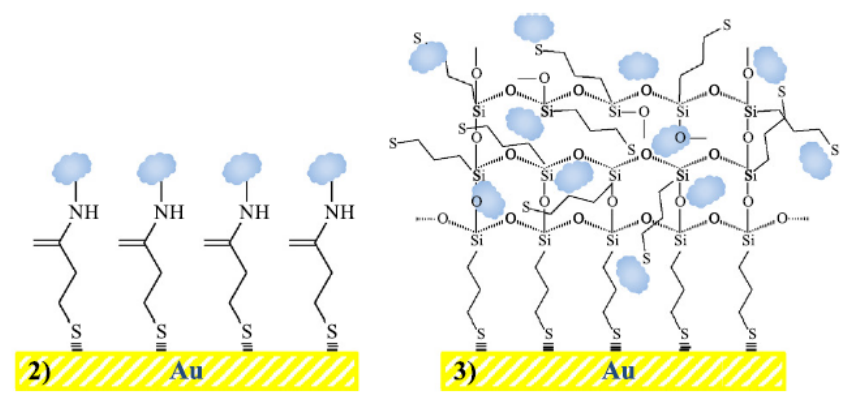

1) Au

2)

B

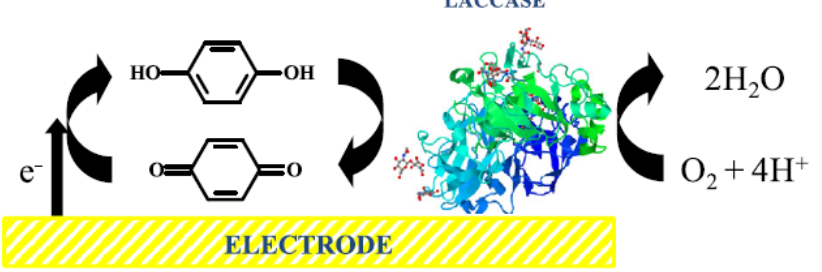

Scheme 1. (A) Enzyme immobilization strategies employed for biosensor fabrication:

1) direct adsorption, 2) covalent binding, 3) sol-gel encapsulation. (B) Catalytic reaction for phenolic compounds.
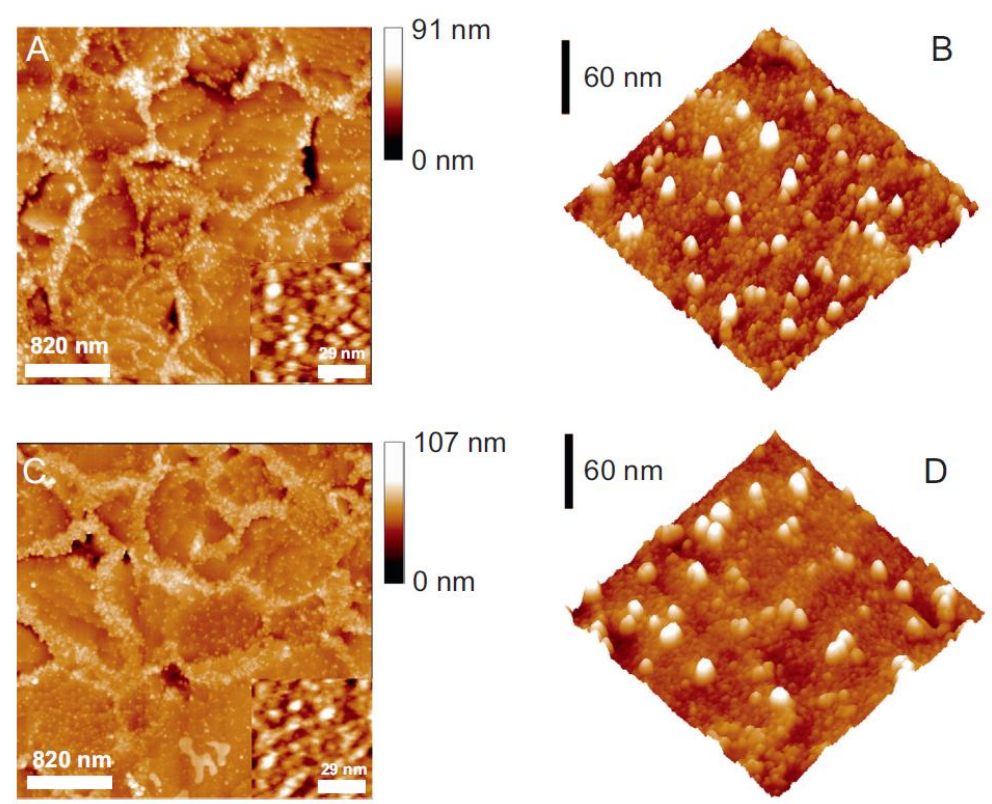

Figure 1. (A) $3.5 \times 3.5 \mu \mathrm{m}^{2}$ and (B) $0.5 \times 0.5 \mu \mathrm{m}^{2}$ tapping-mode AFM images of a TvL layer adsorbed on a gold substrate. Inset of figure A: a higher magnification image ( $75 \mathrm{x}$ $75 \mathrm{~nm}^{2}$ ) of the same system. (C) $3.5 \times 3.5 \mu \mathrm{m}^{2}$ and (D) $0.5 \times 0.5 \mu \mathrm{m}^{2}$ tapping-mode AFM images of a TvL layer covalently bonded to a DTSP-modified gold substrate. 
Inset of figure $C$ : a higher magnification image $\left(75 \times 75 \mathrm{~nm}^{2}\right)$ of the same system. All images have been taken under buffer conditions.
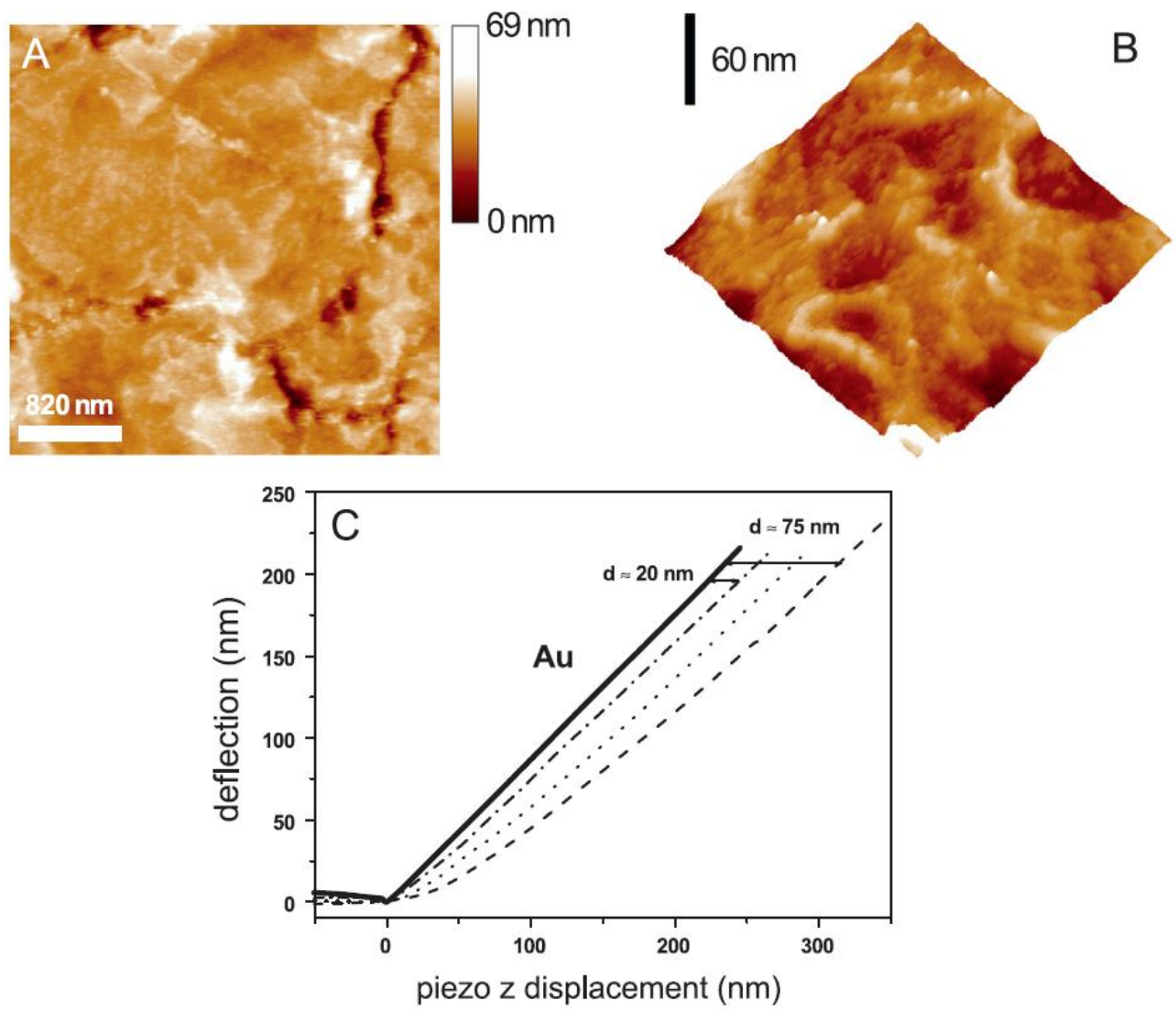

Figure 2. (A) $3.5 \times 3.5 \mu \mathrm{m}^{2}$ and (B) $0.5 \times 0.5 \mu \mathrm{m}^{2}$ tapping-mode AFM images of MPTS/Au, taken under buffer conditions. (C) Force curves taken in air with the same silicon tip on the gold (solid bold line) and MPTS/Au systems. The curves corresponding to the MPTS/Au (dash-dotted, dotted and dashed lines) have been taken at different sample locations. The thickness of the polymer layer varies in the $20-75 \mathrm{~nm}$ range. 

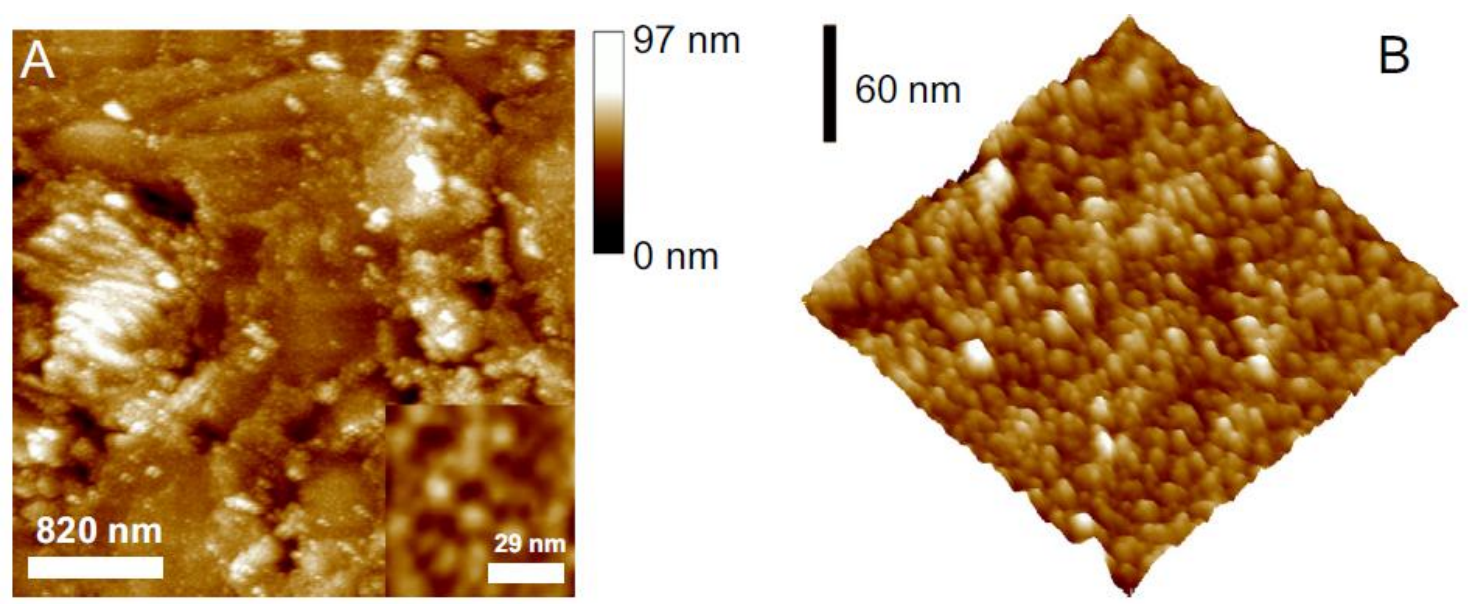

Figure 3. (A) $3.5 \times 3.5 \mu \mathrm{m}^{2}$ and (B) $0.5 \times 0.5 \mu \mathrm{m}^{2}$ tapping-mode AFM images of TvL/MPTS/Au. Inset of figure A: a higher magnification image $\left(75 \times 75 \mathrm{~nm}^{2}\right)$ of the same system. All images have been taken under buffer conditions.
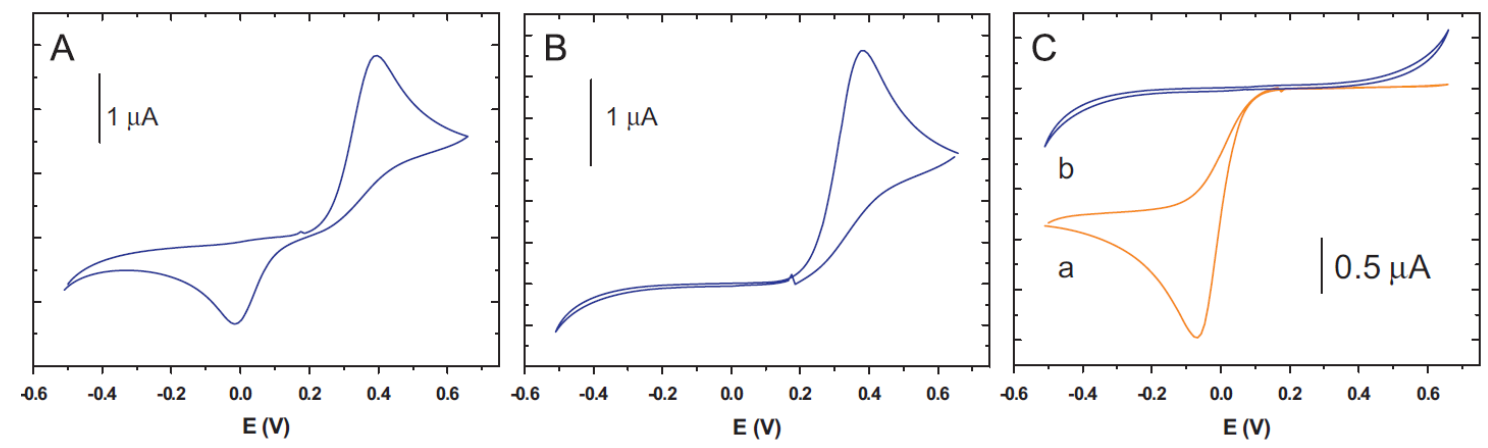

Figure 4. Cyclic voltammetric response for a bare gold electrode $(\mathrm{Au})$ in $0.1 \mathrm{M}$ acetate buffer ( $\mathrm{pH} 4.5$ ) solution containing $100 \mu \mathrm{M}$ hydroquinone cycling the potential from $+0.18 \mathrm{~V}$ towards positive (A) or negative values (B). (C) Cyclic voltammetric response for TvL/Au biosensor immersed in $0.1 \mathrm{M}$ acetate buffer solution in the presence (a) and in the absence (b) of $100 \mu \mathrm{M}$ hydroquinone. The scan rate was $10 \mathrm{mV} / \mathrm{s}$. 
Figure 1S. Successive voltammetric response for TvL/Au (A), TvL/DTSP/Au (B) and TvL/MPTS/Au (C) biosensors immersed in $0.1 \mathrm{M}$ acetate buffer solution in the presence of $100 \mu \mathrm{M}$ hydroquinone. The scan rate was $10 \mathrm{mV} / \mathrm{s}$.

Figure 2S. Chronoamperometric measurements for TvL/Au biosensor at an applied potential of $-0.1 \mathrm{~V}$ in a $0.1 \mathrm{M}$ acetate buffer solution for increasing HQ concentrations.

Figure 3S. Calibration plot of current response versus hydroquinone concentration in a 0.1 $\mathrm{M}$ acetate buffer solution (obtained from chronoamperometric measurements at an applied potential of $-0.1 \mathrm{~V}$ ) for TvL/Au (A), TvL/DTSP/Au (B) and TvL/MPTS/Au (C) biosensors.

Table 1. Analytical properties of several laccase-based biosensors reported in the literature, including the present work. 